\title{
Energy-Efficient and Fault-Tolerant Positioning of Multiple Base Stations
}

\author{
Soo Kim, JeongGil Ko, Jongwon Yoon, and Heejo Lee \\ Korea University \\ \{sooo,jgko, yoonj, heejo\}@korea.ac.kr
}

\begin{abstract}
As the nodes have limited battery power in Wireless Sensor Networks (WSNs), energy efficiency and fault tolerance should be the two major issues in designing WSNs. However, previous studies on positioning base stations (BSs) in WSNs are focused on energy efficiency only. Yet, mission-critical applications like emergency medical care systems should be guaranteed continuous services considering fault tolerance. In this paper we propose to place multiple BSs considering not only energy efficiency but also fault tolerance. We present two strategies to find the optimal position of BSs; (1) minimizing the average transmission energy for energy efficiency; and (2) minimizing additional energy consumption after a BS failure for fault tolerance. The optimal positions for multiple BSs are derived by the metric that considers both energy efficiency and fault tolerance, with a weight factor. Our simulation results show that fault tolerance is important and strongly related to elongation of network lifetime. In addition, we show that our proposed scheme is more energy-effective than previously suggested strategies on unexpected environmental changes which occur commonly in WSNs and sustain the network lifetime effectively under BS failures.
\end{abstract}

\section{Introduction}

Wireless sensor networks (WSNs) consist of a large number of sensors, which are small devices with sensing, processing and transmitting capabilities with limited power resources. Sensors in WSNs monitor a region and transmit information to the base stations (BSs) via wireless channels. The communication between sensors and BSs can be either direct (single-hop) or multi-hop. The energy consumption of each sensor is strongly related to the distance between the a sensor and a BS. Recent studies for energy efficiency in WSNs are mostly focused on minimizing the transmission distance by enhancing routing protocols or utilizing mobility of devices in WSNs. Recently, there have been several studies on BS placement for energy efficiency. Gandham et al. introduced the importance of energy efficient BS placement [1]. Vaas et al. presented the idea of moving the BS of a sensor network, in order to decrease the amount of energy required for communication [2].

Fault tolerance is also a critical issue in designing WSNs. Due to the limited battery and a hostile environment of WSNs, sensors and base stations are vulnerable and can be frequently inactive. Inactive devices increase the energy consumption of numerous

\footnotetext{
* Corresponding author.
} 
sensors and eventually decrease the network lifetime; it discontinues the services in a network at all. However mission-critical applications like the emergency medical care or national defense should be guaranteed with continuous services, minimizing failures of BSs and sensors. Especially, failures on BSs give much more impact to a network than failures on sensor nodes. Zimmermann et al. emphasized that a BS can be faulty and the BS failure can severely degrade the performance of WSNs [3].

In this paper we propose the positioning of multiple BSs considering both energy efficiency and fault tolerance. We use minavg, minimizing the average energy consumption, for the measurement of energy efficiency. This strategy is proven to be the best choice of three strategies by experiments in [2]. In addition, to consider fault tolerance of a WSN, we propose a new key-point to minimize additional energy consumption after a base station failure. Failure on a BS brings much more effects to a WSN than failure on a sensor, therefore we focus on BS failure to measure fault tolerance. The difference between our work and previous studies considering both energy efficiency and fault tolerance [4,5,6] is that we find the optimal placement of multiple BSs, while the authors of [4,5,6] try to enhance routing protocols of WSNs.

The main contribution of our proposed scheme is guaranteeing the elongation of the network lifetime in WSNs, while regarding the failure of BSs. Our simulation results show that using our scheme, which balances energy efficiency and fault tolerance, can sustain the network lifetime twice more than using the scheme considering only energy efficiency.

\section{System Model}

\subsection{Assumptions}

Before we describe the system model, we state our assumptions for this work.

- We assume that the sensors are randomly distributed, on a $n \times n$ region.

- There are multiple sink nodes in the network, which are called base stations (BSs). We assume that base stations do not have limited energy constraints.

- The sensors are static and have energy constraints.

- Only a single device may occupy a single x-y coordinate.

- The sensors operate in an event-driven way.

- The time is split into equal periods and we assume that an event can be reported only at the beginning of a time period.

- To calculate the transmission energy between a sensor and a BS, we assume that the sensors communicate with the BS directly, i.e. single-hop.

- The energy used for communication is proportional to $d^{\alpha}$, where $d$ is the transmission distance and $\alpha$ is the attenuation parameter, typically between 2 and 4. [2]

- Although sensing requires additional energy, this is far less than the energy used in communication; thus we neglect it. [2]

\subsection{Minimizing Average Energy Consumption}

Vass et al. proposed three strategies for positioning BSs: minavg, minmax and minrel [2]. The minavg strategy is to minimize the average (total) energy consumption using the distance between sensor nodes and BSs, the minmax strategy is to minimize the 


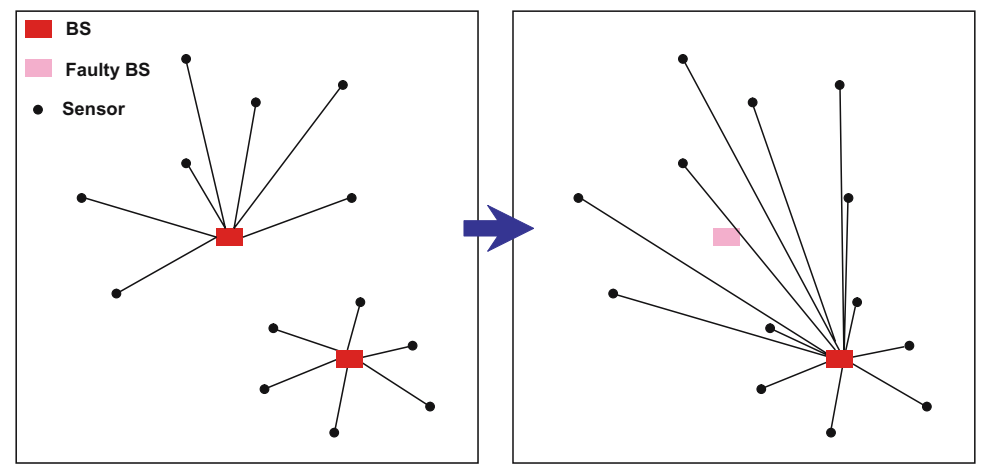

Fig. 1. Additional energy consumption caused by the failure of a BS

transmission energy for the most remote sensor in the network, and the minrel strategy is to minimize the maximum relative energy that a sensor node has to spend on transmission. Through the experimental results of these three strategies in [2], the minavg strategy is proved to be the best strategy for prolonging the network lifetime. Therefore we use the minavg strategy when considering energy efficiency. Let $V$ denote the set of all sensors, and $A \in V$ the set of active sensors. Let $(x, y)$ denote the coordinates of the $\mathrm{BS}$, and $\left(x_{i}, y_{i}\right)$ the coordinates of the $i$ th sensor $(i \in V)$. The energy needed for node $i$ to transmit data is

$$
E_{i}=E_{0}\left(\left(x-x_{i}\right)^{2}+\left(y-y_{i}\right)^{2}\right)^{\alpha / 2},
$$

where $E_{0}$ is a constant and $\alpha$ is the attenuation parameter. The energy consumed by all the active sensors is $E=\frac{1}{n(A)} \sum_{i \in A} E_{i}$, here $n(A)$ is the number of active sensors. It is clear that the optimal location which makes $E$ the smallest is

$$
\left(x_{0}, y_{0}\right)=\arg \min _{(x, y)} E .
$$

In a WSN with the region of $n \times n$, where the sensors are uniformly deployed, the optimal location for the initial BS is $\left(\frac{1}{n}, \frac{1}{n}\right)$. However, there is no closed solution for Eq. (2) since it depends on the network topology. Therefore, it should be solved using optimization methods.

\subsection{Minimizing Additional Energy Consumption after BS Failure}

We now measure the tolerance of a WSN against faults on BS. Although failure on a sensor node also effects the a network, this is far smaller than the effect by failure on a BS; thus, we only consider BS failure. There are various factors that make a BS faulty: energy depletion, attacks on purpose or natural disasters. Fig 1 shows the effect of BS failure in a WSN with two BSs. It is clear that the average distance between each sensor node and its nearest BS increases after a fault. It shows that failure on a BS increases total energy consumed in the network and eventually decreases the total network lifetime. Thus, a network is fault-tolerant if the difference between a network 
before the occurrence of faults and a network after the occurrence after faults is small. Additional energy consumption after faults on $f(0 \leq f \leq k)$ BSs is

$$
E_{f}=\max E^{\prime}(f)-E,
$$

where $E^{\prime}(f)$ is the average energy consumption of all active sensors when $f$ BSs are faulty. Since $E_{f}$ varies to the position of faulty BSs, we choose the maximum $E^{\prime}(f)$ as the worst case. Eq. (3) is only possible when there are multiple base stations in a network, since with a single $\mathrm{BS}, E_{f}$ will be a value of 0 or $\infty$. Also, $E^{\prime}(f) \geq E$ since routing distances of sensors which have been communicating with BSs that are faulty increases. A network is fault-tolerant if $E_{f}$ is small, since it means the effect of faulty BSs to the energy consumption is low. Therefore, the optimal location for $j$ th base station $(j>1)$ is defined as

$$
\left(x_{j}, y_{j}\right)=\arg \min _{(x, y)} E_{f}
$$

\subsection{Synthesis of Energy Efficiency and Fault Tolerance}

We proposed an energy-efficiency metric $E$ and a fault-tolerance metric $E_{f}$ in the previous subsections. The position that makes $E$ and $E_{f}$ to the minimum is the optimal position for BS positioning. However it is difficult to minimize both two metrics simultaneously. We show this tradeoff in the next section. To solve this problem we sum two metrics using a weight factor $\omega . \Phi(f, \omega)$ is the function reflecting both energy efficiency and fault tolerance with $f$ faulty nodes and weight factor $\omega$. This is defined as,

$$
\Phi(f, \omega)=(1-\omega) \cdot \frac{E-E^{\min }}{E^{\text {max }}-E^{\min }}+\omega \cdot \frac{E_{f}-E_{f}^{\min }}{E_{f}^{\max }-E_{f}^{\min }},
$$

where $f$ is the number of faulty BSs as used in Eq. (3). $E$ and $E_{f}$ are all normalized to $E^{\max }, E^{\min }, E_{f}^{\max }$ and $E_{f}^{\min } . \Phi(f, \omega)$ can be an energy-efficient function when $\omega=0$, a fault-tolerant function if $\omega=1$, or an equally balanced function if $\omega=0.5$. The optimal position of the $j$ th BS with $f$ faulty nodes which is derived from Eq. (5) is

$$
\left(x_{j}, y_{j}\right)=\arg \min _{(x, y)} \Phi(f, \omega) .
$$

\subsection{Scheme for Optimal BS Positioning}

With $\Phi(f, \omega)$ we can derive the optimal position to place a BS. Fig. 2 shows a brief scheme to find the optimal position for the $(k+1)$ th BS. When finding the optimal position of the initial BS, we use $\Phi(0,0)$ since fault tolerance is not considered as previously mentioned. Otherwise we use $\Phi(f, \omega)$, which reflects both energy efficiency and fault tolerance. We search the position for BSs in a greedy method rather than a simultaneous method. This is because there is a big difference in complexity between the two methods. The complexity of the greedy method is $\mathcal{O}(n)$. Yet, the complexity of the simultaneous method is $\mathcal{O}\left(n^{\alpha}\right)$ where $\alpha$ is the number of BSs. This is complexity is much higher compared to the complexity of the greedy method. 
Optimal Positioning Scheme for the $(k+1)$ th BS

$k:$ number of previously deployed BSs

$n:$ width/height of a WSN

$(x, y)$ : optimal placement of the $(k+1)$ th BS

$f$ : number of faulty BSs $(0 \leq f \leq k+1)$

$\Phi_{i j}: \Phi(f, \omega)$ when the $(k+1)$ th BS is placed at $(i, j)$

$\Phi_{\text {temp }}=1, \mathrm{x}=0, \mathrm{y}=0$

if $k=\mathbf{0}$

for $i=1: n$

for $j=1: n$

$\Phi_{i j}=\Phi(0,0)$

if $\Phi_{\text {temp }}>\Phi_{i j}$ then

$\Phi_{\text {temp }}=\Phi_{i j}, x=i, y=j$

endfor

endfor

else (if $k>\mathbf{0}$ )

for $i=1: n$

for $j=1: n$

$\Phi_{i j}=\Phi(f, \omega)$

if $\Phi_{\text {temp }}>\Phi_{i j}$ then

$\Phi_{\text {temp }}=\Phi_{i j}, x=i, y=j$

endfor

endfor

Fig. 2. Pseudocode for the optimal position $(x, y)$ of the $(k+1)$ th BS using $\Phi(f, \omega)$

\section{Simulation Results}

In this section, using our simulations, we show the effectiveness of positioning multiple BSs while considering both energy efficiency and fault tolerance. First, we find the optimal position for the initial BS using $E$, the metric for energy efficiency. Second, we find the optimal position for the second BS using $\Phi(1,0), \Phi(1,0.5)$ and $\Phi(1,1)$, and compare the additional energy consumption by simulating a BS failure. Finally, we compare the energy consumption of a network with three BSs placed with the three different metrics. The network lifetime under different metrics $(\Phi(f, 0), \Phi(f, 0.5)$ and $\Phi(f, 1))$ and the number of faulty BSs $(0 \leq f \leq 2)$ shows the necessity and effectiveness of considering both energy efficiency and fault tolerance, especially in vulnerable network environments.

\subsection{The Optimal Position for the Initial BS}

For simulations, we use a $20 \times 20$ region WSN with 100 sensor nodes deployed at random positions, as shown in Fig 3 As mentioned in the previous section, only energyefficiency is considered in finding the optimal position for the initial BS; $F$ is not capable if a single BS is deployed in a network. The optimal position of the initial BS in our 


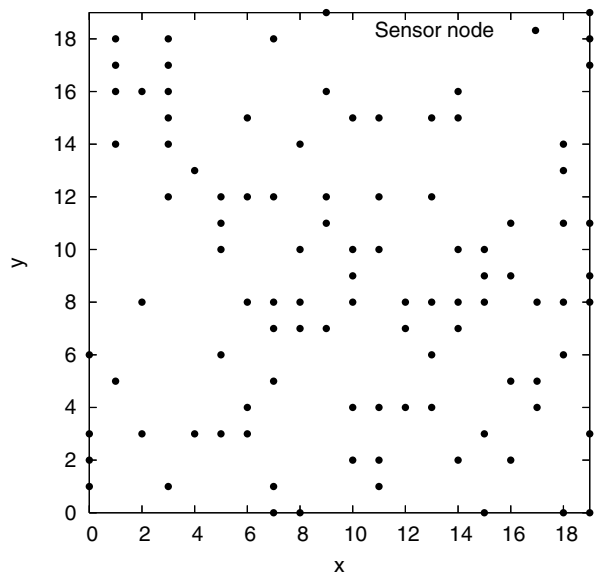

Fig. 3. Deployment of sensors for the simulation

testbed network is $(9,9)$, where $E$ is minimized to the smallest using Eq. (2). If sensor nodes are uniformly deployed in a network, the optimal location for the initial $\mathrm{BS}$ is center of a network.

\subsection{The Optimal Position for the Additional BSs}

Next, we find the optimal position for additional BSs. Unlike the initial BS, here we consider not only energy efficiency but also fault tolerance. We run simulations in three different conditions while varying the weight factor $\omega$; (a) $\Phi(1,0)$ to consider energy efficiency only, (b) $\Phi(1,1)$ to consider fault tolerance only, and (c) $\Phi(1,0.5)$ to consider both energy efficiency and fault tolerant with equal proportion.

Fig 4 (a) shows that two additional BSs are located far from the first BS. This is because placing additional BSs near the initial BS hardly decreases $E$. However in cases like these, the energy consumption can be seriously increased if a failure occurs to any BS, especially the initial BS. In the latter part of this section, we measure and compare the energy consumption of each metric under the occurrence of a BS failure.

On the other hand, positioning the additional BSs near the initial BS makes a network tolerant to BS failure as shown in Fig 4 (b). The positions of two additional BSs that minimize $\Phi(1,1)$ are $(9,8)$ and $(9,10)$, marked as rectangles in Fig 4 (b). This is because the closer the additional BSs gets to the initial BS, the smaller the additional energy consumption gets when a BS faces a fault. However, here the average distance hardly decreases after the positioning of the additional BSs; the advantage of adding more BSs is technically undesirable.

$\Phi(1,0.5)$ reflects energy efficiency and fault tolerance with a balanced ratio of $50 \%$ each. As seen in Fig 4 (c), the distance between the three BSs are shorter than Fig 4 (a) but longer than Fig 4 (b). It shows that $\Phi(1,0.5)$ does not minimize both $E$ and $F_{n}$, is more fault-tolerant than $\Phi(1,0)$ and more energy-efficient $\Phi(1,1)$. 


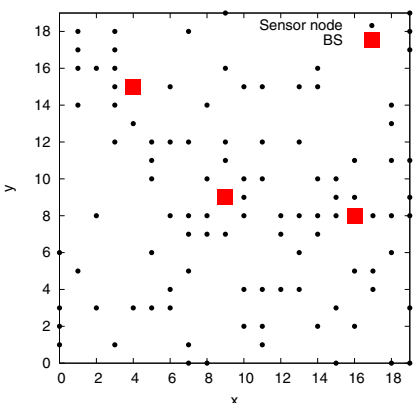

(a) $\Phi(1,0)$ - Energy-efficiency only

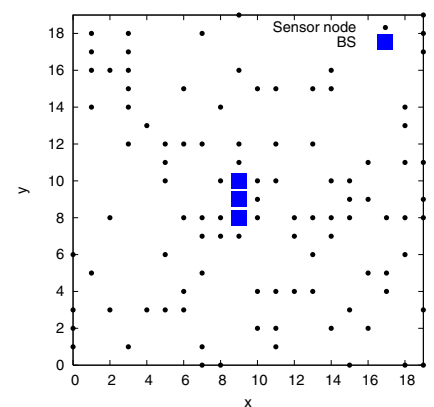

(b) $\Phi(1,1)$ - Fault-tolerance only

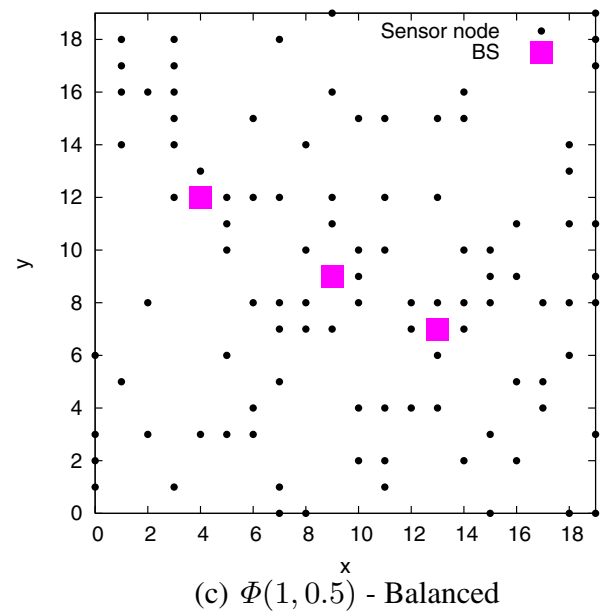

Fig. 4. Optimal positioning of three BSs using $\Phi(1, \omega)$

\subsection{Comparison of the Energy Consumption}

We run simulations for comparing energy consumption for each base station placement algorithm. Each simulation is performed to check the number of sensor nodes that are still actuve in the network per each round. A round is the unit of time and consists of $k$ events. The number of events within a round is modeled as a uniformly-distributed random variable between 0 and $n(A)$, where $n(A)$ is the number of active sensor nodes. A sensor node is randomly chosen to communicate with the nearest BS for every event. Every active sensor sends the same amount of data in a round, and communicate with the nearest BS directly, i.e. singlehop. The initial energy for each sensor is $300 \mathrm{~J}, E_{0}$ is $0.25 \mathrm{~J}$ and the attenuation exponent $\alpha$ is set to 3 .

First, we simulate the energy consumption of the network with a single BS and plot the number of sensor nodes that remain active for 100 rounds in Fig. The first sensor dies in the first round and the end of the 100th round 9 nodes remain alive when the BS is located at the worst position, e.g. where $E$ is maximized (Fig 5(a)). On the other hand, the first sensor dies in the sixth round and more than 28 nodes are alive after 100 rounds if the $\mathrm{BS}$ is located at the optimal position e.g. where $E$ is minimized (Fig 5 (a)). 


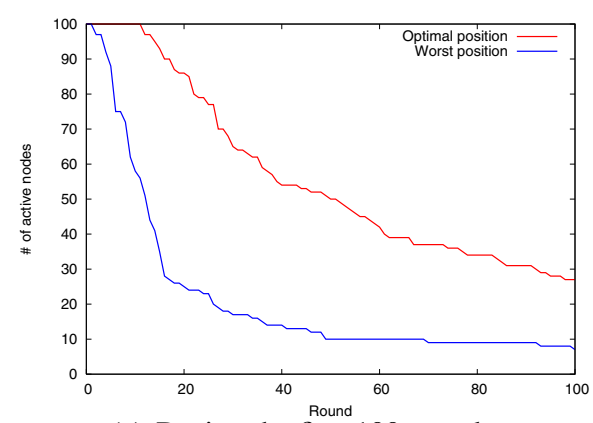

(a) During the first 100 rounds

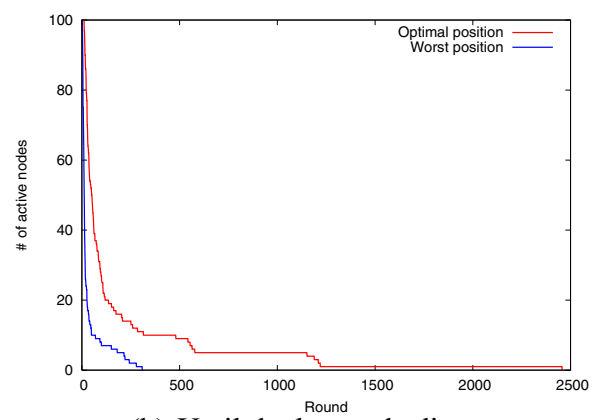

(b) Until the last node dies

Fig. 5. Energy consumption with a single BS

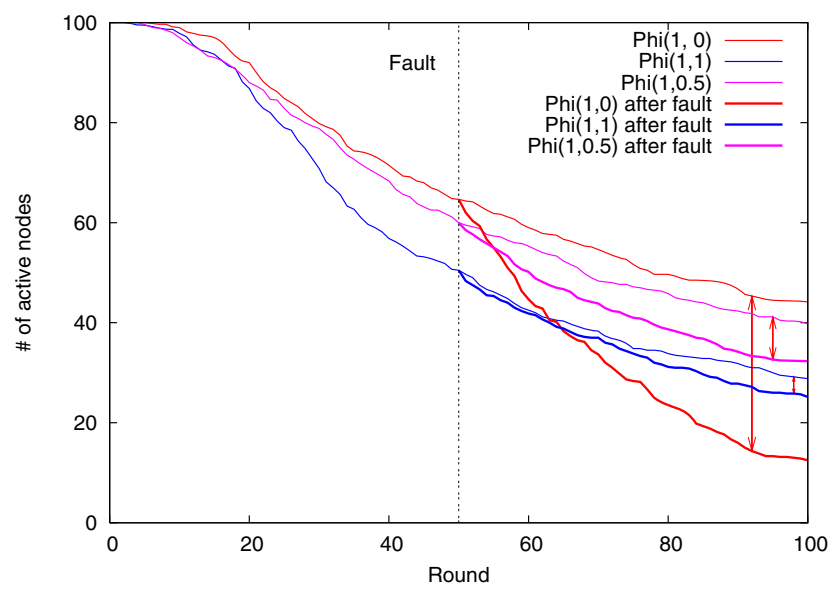

Fig. 6. Energy consumption with 2 BSs, a fault on one BS when round=50

Also when the BS is at its worst position, the last sensor dies within the 300th round. However, the final node dies near the 2500th round when the BS is place at a position with minimum $E$, showing performance of $800 \%$ than when $E$ is maximized (Fig 5 (b)). As expected, placing the first BS to the position where $E$ is minimized decreases the energy consumption of sensors and increases the network lifetime. Through Fig 5 it is seen that considering $E$ as a metric is a necessity.

Second, we simulate the energy consumption of the network with two BSs and plot the number of sensor nodes that remain active for 100 rounds in Fig 6 The simulation environment is equivalent to the simulation done for the network with a single $\mathrm{BS}$ in Fig. 5. However, to measure fault tolerance, we assume there is a BS failure in the 50th round and only one BS can be active from that point. The network with two BSs placed at the $\Phi(1,0)$ position shows the best result among the three conditions when there is no faulty BS in the network (round 0 to 50). This result is what we expected, since the smaller the weight factor $\omega$ becomes, the more energy-efficient the network is. 


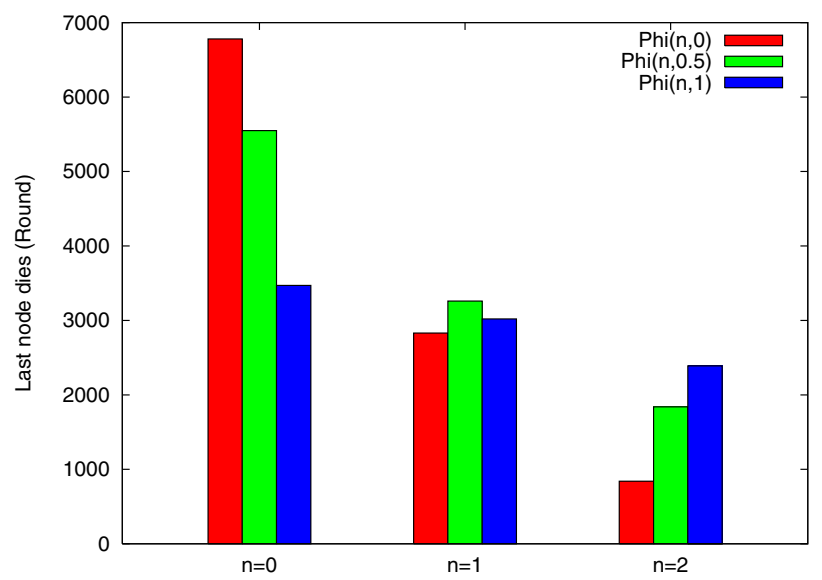

Fig. 7. Network lifetime with three BSs

However, the result becomes quite different when we start to consider faults in BSs. The arrows indicate the difference in the number of active nodes when a network consists of a BS with fault to a network with all BSs active. Since $\Phi(1,0)$ only considers energy efficiency and does not consider fault tolerance at all, $\Phi(1,0)$ shows the widest gap. Consequently when using $\Phi(1,0)$, we can imply that a BS failure in the network can rapidly decrease the network lifetime. The results of $\Phi(1,0)$ gets even worse than the network using $\Phi(1,1)$. On the contrary, the network using $\Phi(1,1)$ is the most fault-tolerant of all; since $\Phi(1,1)$ only considers fault tolerance, despite of the losses in energy efficiency. The network using $\Phi(1,0.5)$ shows moderate decrement in the number of nodes remaining active after a BS failure. The energy consumption without BS failure is not the best of the three, but approximates to the result of $\Phi(1,0)$. Also, the additional energy consumption due to a BS failure shows far better performance than the result of $\Phi(1,0)$. Although the numbers fall it still remains higher than that of $\Phi(1,1)$. Therefore, $\Phi(1,0.5)$ shows the most desired result between the three conditions in the network on a comprehensive basis.

Third, we perform the simulation of energy consumption in the network with three BSs and plot the round when the last node dies, showing the the network lifetime. Fig 7 shows the network lifetime for each metric: $\Phi(f, 0), \Phi(f, 1)$ and $\Phi(f, 2)$. We vary $f$, the number of faulty BSs, from 0 to 2 , to identify the decrement of network lifetime by the number of faulty BSs. When using $\Phi(f, 0)$, the network lifetime for the network without BS faults is the longest compared to the other two cases. Yet, the lifetime decreases rapidly by BS failures. On the contrary when using $\Phi(f, 1)$, the network lifetime without fault is not desirable but fault tolerance is the best of the three metrics. This can be implied from the fact that the graph for $\Phi(f, 1)$ does not change severely in any of the three cases $(f=0, f=1$, and $f=2)$. The balanced metric $\Phi(f, 0.5)$ reduces the additional energy consumption by BS failure and increases the network lifetime better than $\Phi(f, 0) . \Phi(1,0.5)$ improves the network lifetime up to $15 \%$ than $\Phi(1,0)$, and the lifetime of $\Phi(2,0.5)$ is over $200 \%$ of $\Phi(2,0)$. 


\section{Conclusion}

In this paper we propose an energy-efficient and fault-tolerant method in positioning multiple base stations for wireless sensor networks. Through our simulations, we show that by considering both energy-efficiency and fault-tolerance in selecting the optimal position for multiple base stations can increase the total network lifetime. This is compared to other work that have only considered energy-efficiency and fault-tolerance separately. In this work we have used $\omega$ as 0.5 to give equal balance to energy-efficiency and fault-tolerance. Yet, in our future work we plan to find the optimal value for $\omega$. We expect the optimal value to vary in various situations, so many different situations will be considered in our future work. Also we plan to expand our work in a dynamic wireless sensor network where each sensor node has mobility.

\section{Acknowledgement}

This work was supported in part by the ITRC program of the Korea Ministry of Information \& Communications, and grant No. R01-2006-000-10510-0 from the Basic Research Program of the Korea Science \& Engineering Foundation.

\section{References}

1. Gandham, S.R., et al.: Energy efficient schemes for wireless sensor networks with multiple mobile base stations. In: Proc. IEEE GLOBECOM (2003)

2. Vaas, D., Vidacs, A.: Positioning mobile base station to prolong wireless sensor network lifetime. In: Proc. ACM CoNEXT (2005)

3. Zimmermann, K., et al.: Self-management of wireless base stations. In: Proc. IEEE MICMC (2005)

4. Ganesan, D., et al.: Highly resilient, energy efficient multipath routing in wireless sensor networks. In: Proc. ACM MobiHoc (2001)

5. Krishnamchari, B., et al.: The energy-robustness tradeoff for routing in wireless sensor networks. In: Proc. IEEE ICC (2003)

6. Sha, K., et al.: WEAR: A balanced, fault-tolerant, energy-efficient routing protocol for wireless sensor networks. International Journal of Sensor Networks 1(2) (2006) 